\title{
Avian Influenza Surveillance System with Wearable Wireless Sensor Node Using $\mathrm{Pb}(\mathrm{Zr}, \mathrm{Ti}) \mathrm{O}_{3}$ Microcantilever
}

\author{
Hironao Okada*, Hirofumi Nogami, Takeshi Kobayashi and Toshihiro Itoh \\ National Institute of Advanced Industrial Science and Technology \\ 1-2-1 Namiki, Tsukuba, Ibaraki 305-8564, Japan
}

(Received June 10, 2013; accepted August 21, 2013)

Key words: health monitoring, activity, low power consumption, wireless sensor network

We report an avian influenza surveillance system with wireless sensor nodes with which the activity of chickens can be continuously monitored. Intermittent measurement is effective in decreasing the power consumption of a wireless sensor node. However, the measurement interval must be short enough to obtain accurate data in activity measurement of a chicken. In this case, the power consumption of the node is increased owing to the huge amount of transmission data. In this research, we have developed and characterized a method of detecting an anomalous state of a chicken for the continuous activity monitoring with a small amount of transmission data. The result of the simulation using data from infection experiments indicated that the average detection time was about $8 \mathrm{~h}$ before death and the necessary number of transmissions per hour was 33.

\section{Introduction}

Ubiquitous sensor networks that consist of many wireless sensor nodes distributed in our surroundings and linked together are expected to be used in health and medical monitoring applications ${ }^{(1,2)}$ as well as those of environmental monitoring, control, and security. As an application of wireless sensor networks, our group has been developing a global avian influenza surveillance system by monitoring the health of chickens with wireless sensor nodes in poultry farms. (3) The highly pathogenic avian influenza (HPAI) virus (H5N1) infection in birds has continued, and has acquired pathogenicity not only in birds but also in mammals. The more cases of infected migratory birds and domestic fowls increase, the more human cases increase and the variation of the virus progresses. Consequently, the risk of the occurrence of a pandemic flu with transmissibility among humans increases. Therefore, a global avian influenza surveillance system for the earlystage detection of infected bird cases must be effective to defend human beings from an influenza pandemic.

*Corresponding author: e-mail: hironao.okada@aist.go.jp 
The concept of our avian influenza surveillance system is initial diagnosis by examining the body temperature and activity of chickens. Figure 1 shows this system. The wireless sensor nodes are attached to several chickens. When the surveillance system detects an anomalous state of the chickens, the system automatically alerts administrators through the internet.

Previously, infection experiments were carried out using a prototype of wearable wireless sensor nodes that transmitted body temperature and activity data of chickens every $20 \mathrm{~s}$. A method for detecting the anomalous state of a chicken at an early stage was proposed, and how fast the anomalous state can be detected by the method was examined. It was found that an unusual state could be detected several hours before death.(4) However, the lifetime of the prototype wireless sensor node used in the experiment was about 2 weeks. In this system, it is necessary for the nodes to work for two consecutive years or longer without battery replacement, because the period during which chickens lay eggs productively is about $500 \mathrm{~d}$ and replacing the battery of several thousand or more nodes is not practical. In addition, since the weight of the wireless sensor node including a battery should be less than $1 \mathrm{~g}$, a small button battery must be used. If the transmission interval is increased to reduce the power consumption, the necessary data may be missed. To decrease the power consumption, the measurement method must be fundamentally changed.

We have already reported a method in which continuous activity is measured with a piezoelectric microcantilever ${ }^{(5)}$ and a custom LSI. ${ }^{(6)}$ The piezoelectric microcantilever, which generates voltage as a result of the chicken's movements, is used as an activity sensor, and an embedded comparator with ultralow power consumption in the LSI is utilized for the input circuit of the cantilever. In this work, we have clarified the detection time for the method of continuous activity measurement by simulation and how often the node must transmit the data to realize early detection.

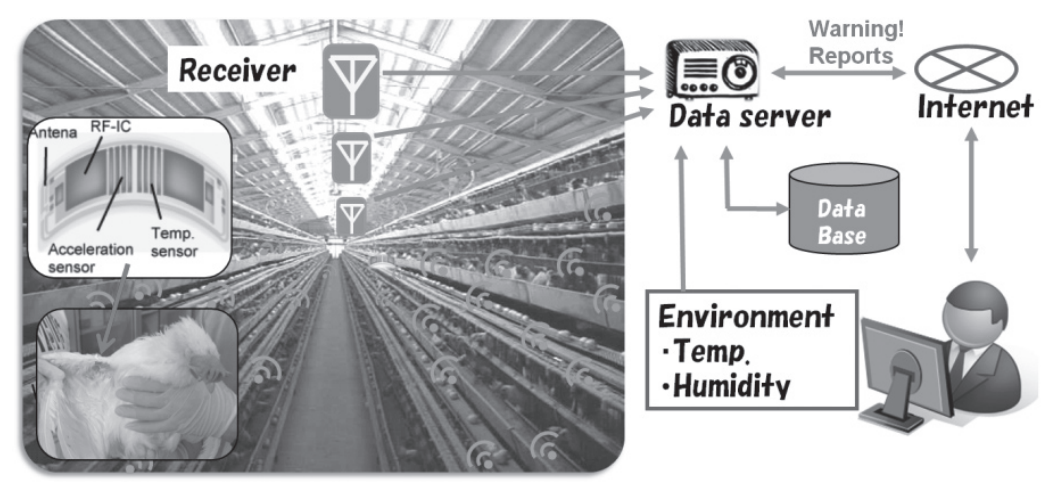

Fig. 1. Image of the avian influenza surveillance system with wireless sensor nodes. 


\section{Architecture of Wireless Sensor Node}

Figure 2 shows the block diagram of a wireless sensor node that can continuously measure activity. The piezoelectric microcantilever and the comparator with several tens of $\mathrm{nA}$ of current consumption make it possible to realize continuous measurement for more than 2 years using a small button battery. In this system, basically, a transmission event occurs when the accumulated number of chicken movements with acceleration exceeding a threshold value $\left(T h_{\mathrm{A}}\right)$ surpasses a preset number $\left(T h_{\mathrm{N}}\right) .^{(6)}$ Before the transmission event, the body temperature is measured using a thermistor. ${ }^{(7)}$ In this system, if the chicken is healthy and active, the transmission events frequently occur. On the other hand, if the chicken is sleeping or in an anomalous state, the number of transmissions decreases. Since $T h_{\mathrm{N}}$ is already known, the activity data need not be included in the transmitted packet. The amount of activity can be deduced from the number of received transmissions per unit time. ${ }^{(8)}$ This can be a useful method for nodes that cannot transmit long packets owing to limitations of the power source.

The most distinct point from the prototype wireless sensor node is the activity measurement. In this architecture, the activity of the chicken can be continuously monitored and the output value is $T h_{\mathrm{N}}$, not the value of acceleration. Figure 3(a) shows an example of the body temperature and jerk of a chicken in the infection experiment. To eliminate the effect of gravity, jerk was calculated from acceleration measurements. ${ }^{(4)}$ It was found that the activity of the chicken decreased several tens of hours before death. Figure 3(b) shows the histogram of jerk in Fig. 3(a) at the time of infection (84-108 h) and for the healthy state $(12-36 \mathrm{~h})$. Although some chickens showed a distinctive

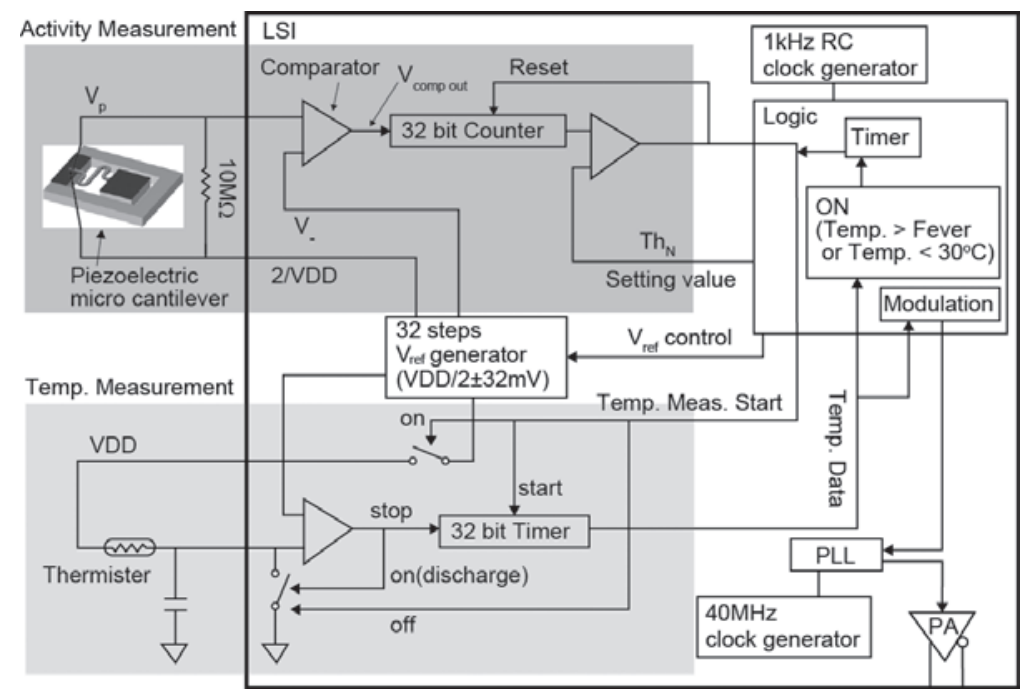

Fig. 2. Block diagram of the wireless sensor node. 


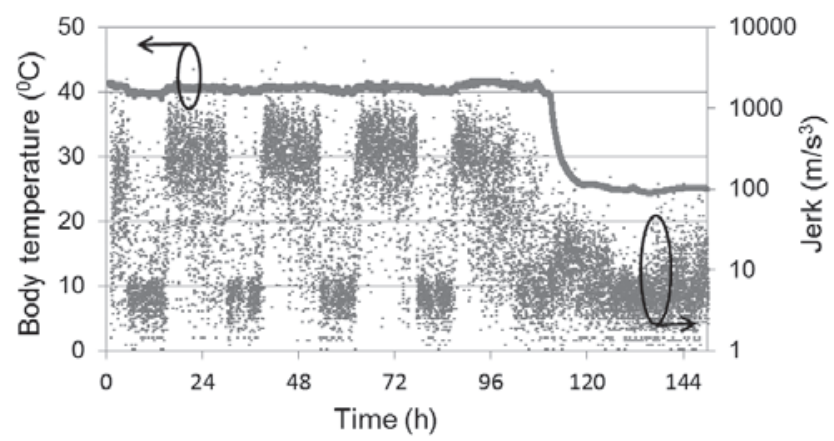

(a)

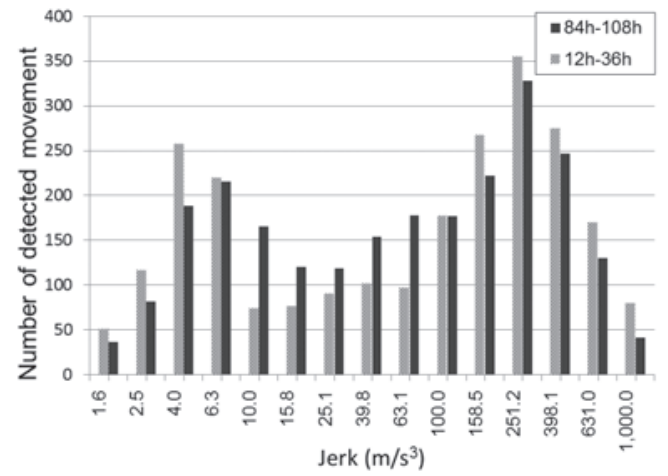

(b)

Fig. 3. (a) Example of body temperature and jerk of a chicken in the infection experiment. (b) Histogram of jerk in (a) at the time of infection $(84-108 \mathrm{~h})$ and in the healthy state $(12-36 \mathrm{~h})$.

difference in the amount of activity between the time of infection and the healthy state, some chickens indicated small changes, as shown in Fig. 3(b). In the case of intermittent operation, the histogram indicates the probability at which each value of jerk can be measured. Thus, if the measurement frequency is decreased, the shape of the histogram can be changed. This can cause delay of detection or false detections, especially for chickens that show a small change of activity. Actually, if the number of data values obtained in the infection experiment was reduced by half in the simulation, several hours of delay occurred. On the other hand, if the activity is continuously measured, this problem does not occur.

\section{Simulation}

In order to clarify how fast we can detect the anomalous state of the chicken using the wireless sensor node with the new architecture, a simulation was carried out. The data of body temperature and jerk obtained from 14 chickens infected with the HPAI 
virus, A/chicken/Miyazaki/K11/2007(H5N1) or CkMZ11,(9) were used in this simulation. The data had to be converted into data that could be obtained using the new architecture. Figure 4 shows the data to which those in Fig. 3(a) were converted. The vertical axis indicates the number of movements with jerk of over $60 \mathrm{~m} / \mathrm{s}^{3}$ per hour ${ }^{(4)}$. The criteria of this detection method are as follows.

$$
\begin{gathered}
\text { Body temperature }>42{ }^{\circ} \mathrm{C} \\
\left(D i f_{\mathrm{t}}>T h_{\mathrm{D}}\right) \text { and }\left(D i f_{\mathrm{t}-1}>T h_{\mathrm{D}}\right) \text { and }\left(\operatorname{Dif}_{\mathrm{t}-2}>T h_{\mathrm{D}}\right) \\
\operatorname{Dif} \mathrm{f}_{\mathrm{t}}=\operatorname{Min}\left(\left|N_{\mathrm{t}}-N_{\mathrm{t}-26}\right|,\left|N_{\mathrm{t}}-N_{\mathrm{t}-25}\right|,\left|N_{\mathrm{t}}-N_{\mathrm{t}-24}\right|,\left|N_{\mathrm{t}}-N_{\mathrm{t}-23}\right|,\left|N_{\mathrm{t}}-N_{\mathrm{t}-22}\right|\right)
\end{gathered}
$$

Death of the chicken was defined as the state in which the body temperature became lower than $30{ }^{\circ} \mathrm{C}$. The detection time indicates how fast we can detect an anomalous state before the time of death. Since the basal body temperature of a chicken is about 41 ${ }^{\circ} \mathrm{C}$, we set the threshold temperature of fever to $42{ }^{\circ} \mathrm{C}$. $N_{\mathrm{t}}$ is the number of movements per hour with more than $60 \mathrm{~m} / \mathrm{s}^{3}$. Th $h_{\mathrm{D}}$ is the threshold value to check the difference in the amount of chicken activity between the present and $24 \mathrm{~h}$ before $\left(\operatorname{Dif}_{\mathrm{t}}\right)$. Since the daily activity of a chicken was not always the same, the activities before and after 2 hours were also compared. In addition, to avoid false detection, especially at the transition between active time and rest time, it was checked if the anomalous state persisted for 3 $\mathrm{h}$ continuously. Thus, if the activity of a chicken is unusual for at least $3 \mathrm{~h}$, the chicken is judged to be in an anomalous state by this system. If $T h_{\mathrm{D}}$ is small, a small amount of change can be detected. Thus, the best value is minimum $D i f_{\mathrm{t}}$ without false detections.

Table 1 shows the results. The number of chickens detected on the basis of fever was 5 , of which 3, however, were identified faster than on the basis of activity. The average detection time was $14.8 \mathrm{~h}$. The CkMZ11 virus did not cause very high fever. ${ }^{(10)}$ On the other hand, the activity of the infected chicken must decrease. It was found that the optimum $T h_{\mathrm{D}}$ was 12, and the average detection time by activity measurement was $8.14 \mathrm{~h}$. The fastest and slowest detections were $22 \mathrm{~h}$ before and $2 \mathrm{~h}$ after death, respectively.

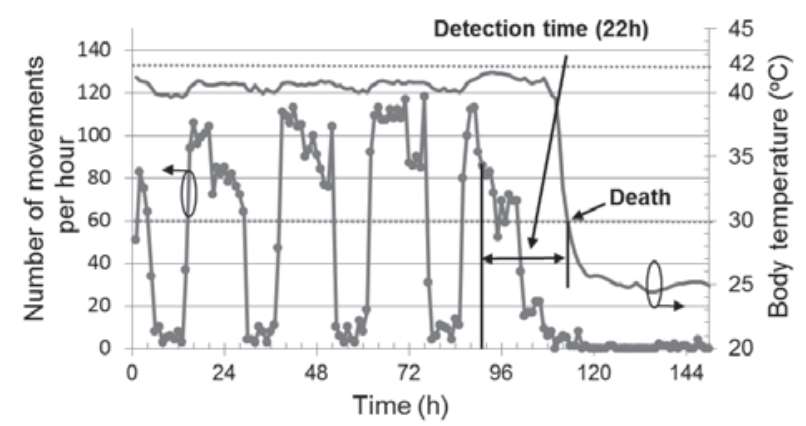

Fig. 4. Converted data of those from the infection experiments. 
Table 1

Result of the simulation.

\begin{tabular}{lcccc}
\hline & \multirow{2}{*}{ Number of detected chickens } & \multicolumn{3}{c}{ Detection time (h) } \\
\cline { 3 - 5 } & & Slowest & Average & Fastest \\
\hline Activity & 14 & -2 & 8.14 & 22 \\
Body temperature & $5(3)^{*}$ & 9 & 14.8 & 18 \\
\hline
\end{tabular}

${ }^{*}$ Three detections based on body temperature are faster than by activity.

\section{Experimental Results and Discussion}

Figure 5 shows the wing-band-type wireless sensor node with the new architecture. A wing band made from an aluminum plate is usually used for the identification of chickens and is affixed to the thin skin at the arm of the chicken. In this node, a U-shaped pin is used to fix the node to a chicken's arm as shown in Fig. 5. In order to examine the optimum value of $T h_{\mathrm{D}}$, which does not cause false detection in the healthy state, for data obtained using this node, we attached the nodes to chickens in a chicken house. This chicken house is not a special facility for infection experiments. The threshold voltage for the piezoelectric microcantilever was configured to change the output of the comparator if a chicken's movement with more than $0.01 \mathrm{~m} / \mathrm{s}^{2}\left(T h_{\mathrm{A}}\right)$ was applied in the node. The value of $0.01 \mathrm{~m} / \mathrm{s}^{2}$ is similar to the converted value of $60 \mathrm{~m} / \mathrm{s}^{3}$. The $60 \mathrm{~m} / \mathrm{s}^{3}$ can be converted to $0.008 \mathrm{~m} / \mathrm{s}^{2}$ by means of multiplication by the measurement time of $140 \mu \mathrm{s} .{ }^{(4)}$ The threshold number $\left(T h_{\mathrm{N}}\right)$ used for the comparison of the number of chicken movements with acceleration of more than $T h_{\mathrm{A}}$ was set to 100 .

Figure 6 shows an example of the data obtained from the nodes over a period of $6 \mathrm{~d}$. During the daytime, the number of transmissions received was about 100 and saturated. The wireless sensor node was configured not to transmit data continuously owing to the limitation of the power source. In this experiment, the time was set to about $40 \mathrm{~s}$. It was found that there was a possibility that the threshold value of acceleration is smaller than 0.01 $\mathrm{m} / \mathrm{s}^{2}$ because the number of movements obtained during rest time is greater than the data in the infection experiment.

We carried out the simulation using the above criteria and the obtained data. It was found that false detection did not occur even when $T h_{\mathrm{D}}$ was decreased to 6 . This result indicates that the data obtained with the developed wireless sensor nodes is more stable than the data in the infection experiment. The infection experiment was carried out in a special facility of biosafety level 3. ${ }^{(9)}$ Although the environmental difference might have caused the difference in $T h_{\mathrm{D}}$, there is also the possibility that the continuous activity measurement increased the stability. The small $T h_{\mathrm{D}}$ makes it possible to detect small changes in activity.

If the transmission frequency is decreased, the power consumption of the wireless sensor node can be decreased. Because every obtained data value indicates the same meaning, that is, there are 100 occurrences of movement with acceleration exceeding $T h_{\mathrm{A}}$, the number of data values received per hour can be decreased by increasing $T h_{\mathrm{N}}$. 


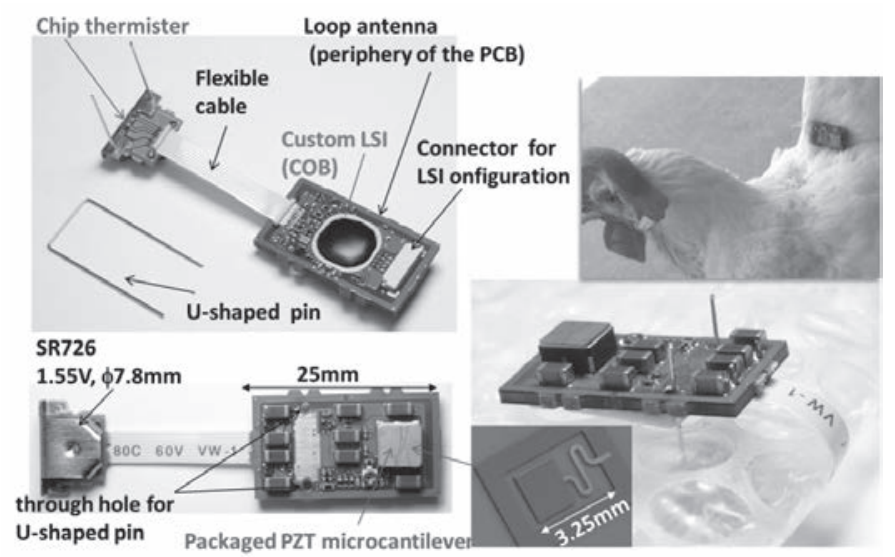

Fig. 5. Wing-band-type wireless sensor node with the new architecture.

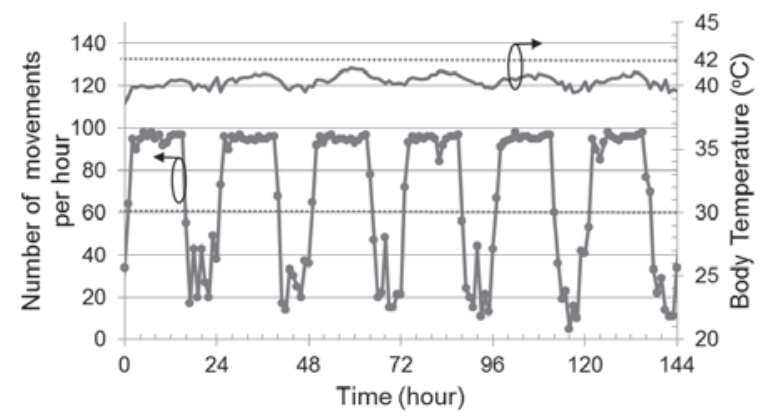

Fig. 6. Example of data obtained using the developed node for $6 \mathrm{~d}$.

Six of $T h_{\mathrm{D}}$ means a difference of 600 occurrences of movement. Thus, although $T h_{\mathrm{N}}$ can be increased to 600 , we thought that half this value (300) might be necessary considering packet loss. In this case, 33 transmissions per hour are required.

\section{Conclusion}

We characterized the detection method for the new architecture of the sensor node in the avian influenza surveillance system and found that the average detection time was about $8 \mathrm{~h}$ before death. In the simulation, the optimized $T h_{\mathrm{D}}$ was 12 . We also demonstrated the method using the developed wing-band-type wearable wireless sensor node. The optimized value of $T h_{\mathrm{D}}$ was 6 . In this case, the number of transmissions per hour can be decreased to 33 . 


\section{Acknowledgements}

This research is funded through a grant from the Core Research for Evolutional Science and Technology (CREST) of Japan Science and Technology Agency (JST) and the Japan Society for the Promotion of Science (JSPS) through the "Funding Program for World-Leading Innovative R\&D on Science and Technology (FIRST Program)", initiated by the Council for Science and Technology Policy (CSTP).

\section{References}

1 S. G. Taylor, K. M. Farinholt, E. B. Flynn, E. Figueiredo, D. L. Mascarenas, E. A. Moro, G. Park, M. D. Todd and C. R. Farrar: Meas. Sci. Technol. 20 (2009) 1.

2 N. Najafi and A. Ludomirsky: Biomedical Microdevices 6 (2004) 61.

3 http://www.aist.go.jp/aist_e/research_results/publications/synthesiology_e/vol3_no3/ vol03_03_p224_p233.pdf.

4 H. Okada, K. Suzuki, K. Tsukamoto and T. Itoh: Proc. Design Test Integration and Packag. of MEMS/MOEMS 2010, p. 253.

5 T. Kobayashi, H. Okada, V. Z. Gang, R. Maeda, T. Masuda and T. Itoh: Proc. Transducers 2011, p. 1014.

6 H. Okada, T. Masuda and T. Itoh: Proc. IEEE Sensors 2011, p. 1197.

7 H. Okada, H. Nogami, T. Masuda and T. Itoh: Proc. 2nd Workshop on Design, Control and Software Implementation for Distributed MEMS 2012, p. 90.

8 H. Okada, T. Masuda and T. Itoh: Proc. IEEE Sensors 2012, p. 648.

9 K. Suzuki, H. Okada, T. Itoh, T. Tada and K. Tsukamoto: J. General Virology 91 (2010) 2302.

10 K. Suzuki, H. Okada, T. Itoh, T. Tada, M. Mase, K. Nakamura, M. Kubo and K. Tsukamoto: J. Virology 83 (2009) 7475. 